\title{
On Schertz's class number formula related to elliptic units for some non-Galois extensions
}

\author{
by
}

JiRo Suzuki (Tokyo)

Introduction. The purpose of this paper is to give a refinement of Schertz's class number formula related to elliptic units. We are going to study some non-Galois cases. Let $K / Q$ be a finite non-Galois extension, $k$ be an imaginary quadratic field, and put $L=K k$. We denote by $h$ the class number of $K$ and by $E$ the unit group of $K$. Suppose that $L / k$ is an abelian extension. Then Schertz [6] has shown a class number formula related to elliptic units as follows:

THEOREM (Schertz). Notations being as above, one can construct a group $F$ of certain elliptic units of $K$ such that

$$
c h / h_{0}=\left(E: F E_{0}\right)
$$

with an explicit constant $c$ depending only on the construction of $F$. Here, $h_{0}$ and $E_{0}$ are the class number and the unit group of the maximal absolutely abelian subfield $K_{0}$ of $K$, respectively.

In this formula, $h_{0}$ can be known in various ways because $K_{0}$ is absolutely abelian. But the constant $c$ is much more complicated and not so small in [6]. Now, to know the class number $h$, we shall make $c$ as small and explicit as possible. Then we have the best possible construction of $F$ in Schertz's formula. Namely,

THEOREM 1. Notations being as above, suppose that the Galois closure L of $K$ is dihedral over $\boldsymbol{Q}$ and cyclic over $k$. Let $n=[K: Q]$. Then we can construct a group $F$ of certain elliptic units such that

$$
\begin{gathered}
h=(E: F) \quad \text { if } n \text { is odd, } \\
2^{b} h / h_{0}=\left(E: F E_{0}\right) \quad \text { if } n=4 \text { or } 2 l,(2, l)=1 .
\end{gathered}
$$

In (0.3), $K_{0}$ is a quadratic field and $b$ is a computable positive integer.

The proof of Theorem 1 is described in Section 3. For that purpose, we prepare some properties of $\boldsymbol{Z}$-modules in cyclotomic fields in Section 1. Schertz's result above is described in Section 2 precisely. Finally, in Section 4, 
we discuss a few more cases where $L / Q$ is not dihedral. In particular, in the case where $[K: Q]$ is the product of two primes we prove Theorem 2 which, together with Theorem 1 , cempletely give a formula for $n=p q$ with primes $p$, $q$. The class number formula $(0.1)$ is previously studied in detail by $\mathbf{H}$. Hayashi [1], K. Nakamula [2], [3], [4] when $n=3,4,5,6$.

1. Preliminaries on cyclotomic fields. Let $m$ be a positive integer, $>2$. Let $\zeta=\zeta_{m}$ be a primitive $m$ th root of unity and put $Q_{m}=Q(\zeta)$. Let $J$ be the complex conjugation of $\boldsymbol{Q}_{m}$ and let $\boldsymbol{Q}_{m}^{+}$be the fixed field of $\boldsymbol{Q}_{m}$ for $J$. Then $Q_{m}^{+}$is the maximal real subfield of $\boldsymbol{Q}_{m}$. Let $\mathcal{O}$ (resp. $\mathcal{O}^{+}$) be the ring of integers of $\boldsymbol{Q}_{m}$ (resp. $\boldsymbol{Q}_{m}^{+}$), and $D_{m}$ (resp. $D_{m}^{+}$) be the discriminant of $\boldsymbol{Q}_{m}$ (resp. $\boldsymbol{Q}_{m}^{+}$). Let $N$ be the norm on $\boldsymbol{Q}_{m} / \boldsymbol{Q}$. Let $\Phi_{m}(X)$ be the $m$ th cyclotomic polynomial:

$$
\Phi_{m}(X)=\prod_{(a, m)=1}\left(X-\zeta_{a}\right)
$$

Throughout this section, $p$ always denotes a prime number.

Let $n \in Q$. We define the function $\Phi_{n}^{*}$ from $\{ \pm 1\}$ to $Z$ according to the value $\Phi_{n}( \pm 1)$ as follows

$$
\Phi_{n}^{*}( \pm 1)= \begin{cases}\Phi_{n}( \pm 1) & \text { if } n>2, n \in Z \\ 1 & \text { if } n \in Q-Z\end{cases}
$$

When $n=1$ or $2, \Phi_{1}^{*}(1)=\Phi_{2}^{*}(-1)=1$ and $\Phi_{1}^{*}(-1)=\Phi_{2}^{*}(1)=2$.

We recall that $m>2$, then $\Phi_{m}( \pm 1)$ is known as follows:

$$
\Phi_{m}(1)= \begin{cases}p, & m=p^{a}, a \geqslant 1, \\ 1, & \text { otherwise. }\end{cases}
$$

Since $\Phi_{m}(-1)=\prod\left(-1-\zeta_{a}\right)=\prod\left(1+\zeta_{a}\right)$,

$$
\Phi_{m}(-1)= \begin{cases}\Phi_{m / 2}(1) & \text { if } m=2(\bmod 4) \\ \Phi_{m}(1) & \text { if } m=0(\bmod 4) \\ \Phi_{2 m}(1) & \text { if } m= \pm 1(\bmod 4)\end{cases}
$$

Therefore,

$$
\Phi_{m}(-1)= \begin{cases}p, & m=2 p^{a}, a \geqslant 1, \\ 1, & \text { otherwise. }\end{cases}
$$

Note that $N(\zeta-1)=\Phi_{m}(1)$. We shall only use $\Phi_{m}^{*}( \pm 1)$ as the absolute norm of some ideal $\neq(0)$. When we use $\Phi_{n}^{*}( \pm 1)$ as the meaning of some positive integer, we can use $\Phi_{n}( \pm 1)$ instead of $\Phi_{n}^{*}( \pm 1)$, without confusion.

Let $p=(\zeta-1) \mathcal{O}$. Then $p$ is a prime ideal of $\mathcal{O}$ when $m$ is a prime power, otherwise $p=\mathcal{O}$.

The following lemma is known (see Washington [7], Lemma 4.19). 
LEMMA 1. The discriminant of the maximal real subfield $Q_{m}^{+}$is given by

$$
\left|D_{m}^{+}\right|=\left(m^{\varphi(m)} / \prod_{p \mid m} p^{\varphi(m) /(p-1)} \Phi_{m}(1) \Phi_{m / 2}(1)\right)^{1 / 2},
$$

where $p$ runs through all prime divisors of $m$.

Here, the factor $\Phi_{m}(1) \Phi_{m / 2}(1)$ equals $p$ or 4 according as $m$ or $m / 2$ is $p^{a}$ or $2^{a}$, where $p$ is an odd prime. Otherwise, $\Phi_{m}(1) \Phi_{m / 2}(1)=1$.

Let $\zeta_{0}$ be an $m$ th root of unity. We consider a $Z$-module given by the following formula:

$$
M=\sum_{j=1}^{m-1} Z\left(\left(\zeta^{j}-1\right)+\zeta_{0}\left(\zeta^{-j}-1\right)\right) .
$$

Since $m>2, M \neq 0$. Denote by $d(M)$ the absolute value of the discriminant of the $Z$-module $M$. Suppose $\zeta_{0}= \pm 1$.

LEMMA 2. Let $\zeta_{0}=1$. Then $M=p \cap \mathcal{O}^{+}$, and

$$
d(M)=\Phi_{m}(1)^{2}\left|D_{m}^{+}\right| .
$$

Proof. Since $\zeta_{0}=1$, we have $M=\sum_{j=1}^{m-1} Z\left(\zeta^{j}+\zeta^{-j}-2\right)$ from (1.3). We shall prove the above in 3 steps.

1. $M$ is an ideal of $\mathcal{O}^{+}$. Since $\mathcal{O}^{+}=Z\left[\zeta+\zeta^{-1}\right]$ (see [7], Proposition 2.16), the $Z$-module $M$ is an ideal of $\mathcal{O}^{+}$. Indeed,

$$
\left(\zeta+\zeta^{-1}\right)\left(\zeta^{j}+\zeta^{-j}-2\right)=\left(\zeta^{j+1}+\zeta^{-j-1}-2\right)+\left(\zeta^{j-1}+\zeta^{-j+1}-2\right)-2\left(\zeta+\zeta^{-1}-2\right) .
$$

2. $p^{2} \cap \mathcal{O}^{+} \subset M \subset p \cap \mathcal{O}^{+}$. The inclusion $M \subset p \cap \mathcal{O}^{+}$is clear. Since $M$ is an ideal of $\mathcal{O}^{+}$and $\left(\zeta+\zeta^{-1}-2\right) \in M$,

$$
p^{2} \cap \mathcal{O}^{+}=(\zeta-1)^{2} \mathcal{O} \cap \mathcal{O}^{+}=\left(\zeta+\zeta^{-1}-2\right) \mathcal{O}^{+} \subset M .
$$

3. $\boldsymbol{p}^{2} \cap \mathcal{O}^{+}=p \cap \mathcal{O}^{+}$. If $p=\mathcal{O}$ then the equality is trivial. Assume $p \neq \mathcal{O}$, then $p$ is the prime ideal which totally ramifies in $Q_{m} / Q$. Let $p^{+}=p \cap \mathcal{O}^{+}$. Since $\left[Q_{m}: Q_{m}^{+}\right]=2, p^{+} \mathcal{O}=p^{2}$. This implies that $p^{2} \cap \mathcal{O}^{+}=p \cap \mathcal{O}^{+}$. Hence $M=p \cap \mathcal{O}^{+}$. In step 3, we saw that $\left[\mathcal{O}^{+}: p \cap \mathcal{O}^{+}\right]=\Phi_{m}(1)$. Using the formula for the discriminant of an ideal of an algebraic number field, the lemma is proved.

Lemma 3. Let $\zeta_{0}=-1$ and let $m$ be even. Then $M=\left(\zeta-\zeta^{-1}\right) \mathcal{O}^{+}$and

$$
d(M)=\Phi_{m / 2}(1) \Phi_{m / 2}(-1)\left|D_{m}^{+}\right| .
$$

Proof. Since $\zeta_{0}=-1$, we have $M=\sum_{j=1}^{m-1} Z\left(\zeta^{j}-\zeta^{-j}\right)$ from (1.3). Further $\mathcal{O}^{+}=Z\left[\zeta+\zeta^{-1}\right]$. For any integer $k$,

$$
\zeta^{k}-\zeta^{-k}=\left(\zeta-\zeta^{-1}\right)\left(\zeta^{k-1}+\zeta^{-k+1}+\zeta^{k-3}+\zeta^{-k+3}+\ldots\right)+e\left(\zeta-\zeta^{-1}\right)
$$


where $e=1$ or 0 . Therefore $M=\left(\zeta-\zeta^{-1}\right) \mathcal{O}^{+}$. The discriminant of $M$ is given by

$$
d(M)=\left|N\left(\zeta-\zeta^{-1}\right)\right|\left|D_{m}^{+}\right|
$$

and

$$
\left|N\left(\zeta-\zeta^{-1}\right)\right|=\left|N\left(\zeta^{2}-1\right)\right|=|N(\zeta-1) N(\zeta+1)|=\Phi_{m}(1) \Phi_{m}(-1) .
$$

The lemma is proved.

Remark 1. The rank of $p \cap \mathcal{O}^{+}$as $Z$-module is $\varphi(m) / 2$. The set $\left\{\zeta^{j}+\zeta^{-j}-2 \mid 1 \leqslant j \leqslant \varphi(m) / 2\right\}$ is an independent system over $Z$. Furthermore the fact that the set is a basis of $p \cap \mathcal{O}^{+}$is proved in a way similar to the proof of the fact that $\left\{\zeta^{j}+\zeta^{-j} \mid 1 \leqslant j \leqslant \varphi(m) / 2\right\}$ is a basis of $Z\left[\zeta^{1}+\zeta^{-1}\right]$. Similarly, $\left\{\zeta^{j}-\zeta^{-j} \mid 1 \leqslant j \leqslant \varphi(m) / 2\right\}$ is a basis of $\left(\zeta-\zeta^{-1}\right) \mathcal{O}$.

Suppose $\zeta_{0} \neq \pm 1$.

LEMMA 4. Let $\zeta_{0}$ be a primitive $d$-th root of unity, where $d$ is a divisor of $m$ and $d \neq 1,2$. Then there is a $Z$-submodule $M_{0}$ of $M$ such that the discriminant of $M_{0}$ on $\boldsymbol{Q}_{m}^{+}$is given by

$$
d\left(M_{0}\right)=\Phi_{d}(-1)^{\varphi(m) / \varphi(d)} \Phi_{m}(1)^{2}\left|D_{m}^{+}\right| .
$$

Proof. Let $M_{0}=\left(1+\zeta_{0}\right)\left(p \cap \mathcal{O}^{+}\right)$. Then $M_{0}$ is a $Z$-submodule of $M$ because $p \cap \mathcal{O}^{+}=\sum_{j=1}^{m-1} Z\left(\zeta^{j}+\zeta^{-j}-2\right)$ by Lemma 2 and

$$
\left(1+\zeta_{0}\right)\left(\zeta^{j}+\zeta^{-j}-2\right)=\left(\left(\zeta^{j}-1\right)+\zeta_{0}\left(\zeta^{-j}-1\right)\right)+\left(\left(\zeta^{-j}-1\right)+\zeta_{0}\left(\zeta^{j}-1\right)\right) \in M .
$$

The discriminant of $M_{0}$ is given by $d\left(M_{0}\right)=\left|N\left(1+\zeta_{0}\right)\right|\left|d\left(p \cap \mathcal{O}^{+}\right)\right|$. Since $\zeta_{0}$ is a primitive $d$ th root of unity, $\left|N\left(1+\zeta_{0}\right)\right|=\Phi_{d}(-1)^{\varphi(m) / \varphi(d)}$. The lemma is proved.

2. Schertz's results. In this section, we shall describe Schertz's result and give the notations. Using the class field theory, there is a positive integer $f$ such that the ray class field $H_{(f)}$ modulo $(f)$ of $k$ includes $L$. Let $\mathrm{Cl}(f)$ be the ray class group modulo $f$ of $k$. The Artin symbol $\left(c, H_{(f)} / k\right)$ gives an isomorphism from $\mathrm{Cl}(f)$ to the Galois group $G\left(H_{(f)} / k\right)$. Since $f=\bar{f}$, the complex conjugation $c \rightarrow \bar{c}$ is an automorphism of $\mathrm{Cl}(f)$. Using this fact, we can prove the next properties. (But the proof is omitted here, see [6].)

The extension $H_{(S)} / Q$ is Galois, the Galois group $G\left(H_{(f)} / Q\right)$ is the semi-direct product $G\left(H_{(f)} / k\right) \cdot\langle J\rangle$, and $G\left(H_{(f)} / k\right)$ is a normal subgroup of $G\left(H_{(\Omega)} / Q\right)$. Since $J^{-1}\left(c, H_{(f)} / k\right) J=\left(\bar{c}, H_{(f)} / k\right)$, we define $c^{J}$ by $\bar{c}$. Let $U$ be the subgroup of $\mathrm{Cl}(f)$ corresponding to the field $L$. Since $[L: K]=2, U^{J}=U$ can be proved. (See $[6,11]$, pp. 67-68.) Therefore $L / Q$ is Galois. There is an element $c_{0}$ of $\mathrm{Cl}(f) \bmod U$ such that $G(L / K)=\left\langle\left(c_{0}, L / Q\right) J\right\rangle$, the Galois group $G(L / Q)$ is the semi-direct product $G(L / k) \cdot\left\langle\left(c_{0}, L / Q\right) J\right\rangle$, and $G(L / k)$ is a normal subgroup of $G(L / Q)$. For the maximal abelian subfield $K_{0}$ of $K$, the composite $L_{0}=K_{0} k$ is the maximal abelian subfield of $L$. Let $U_{0}$ be the subgroup of $\mathrm{Cl}(f)$ corresponding to $L_{0}$. Then $U_{0}=\left\{c^{1-J} \mid c \in \mathrm{Cl}(f)\right\}$. Let $A=\mathrm{Cl}(f) / U$ and $X$ 
be the character group of $A$. Since $U^{J}=U$, we define the action of the automorphism $J$ on $X$ by $\chi^{J}(c)=\chi(\bar{c})$ for any $\chi \in X$. Let $X_{0}=\{\chi \in X \mid$ $\left.\chi^{J}=\chi\right\}$. Then $X_{0}$ is the character group of $U_{0}$. The classes of characters $W=\left(X-X_{0}\right) / \sim$ are defined by the equivalence relation:

$$
\chi \sim \chi^{\prime} \quad \text { if and only if }\langle\chi\rangle=\left\langle\chi^{\prime}\right\rangle \text { or }\left\langle\chi^{J}\right\rangle=\left\langle\chi^{\prime}\right\rangle .
$$

If $L / Q$ is dihedral, then $\langle\chi\rangle=\left\langle\chi^{\prime}\right\rangle$ is equivalent to $\left\langle\chi^{J}\right\rangle=\left\langle\chi^{\prime}\right\rangle$. Therefore, we assume that $\chi \sim \chi^{\prime}$ satisfies $\langle\chi\rangle=\left\langle\chi^{\prime}\right\rangle$ in this section. Later, we shall treat the case $\left\langle\chi^{J}\right\rangle \neq\langle\chi\rangle$ in Section 4. For any class $\omega$ of $W$, let $m_{\omega}$ be the order of an element of $\omega$. We take the subset $\omega^{\prime}$ of $\omega$ defined by

$$
\omega^{\prime}=\left\{\chi^{j} \mid 1 \leqslant j \leqslant m_{\omega} / 2,\left(j, m_{\omega}\right)=1\right\} .
$$

Then $\omega^{\prime} \cup \omega^{\prime J}=\omega$ and $\omega^{\prime} \cap \omega^{J}=\varnothing$. Put $r_{\omega}=\# \omega^{\prime}=\varphi\left(m_{\omega}\right) / 2$. The rank $r$ of $F$ is found in [6], namely,

$$
r=\sum_{\omega \in W} r_{\omega}+r_{0}, \quad r_{0}=\#\left\{\chi \in X_{0} \mid \chi\left(c_{0}\right)=1, \chi \neq 1\right\} .
$$

For any class $\omega$, let $U_{\omega}, A_{\omega}$ and $k_{\omega}$ be the following:

$$
\begin{gathered}
U_{\omega}=\{c \in \mathrm{Cl}(f) \mid \chi(c)=1 \text { for any } \chi \in \omega\}, \\
A_{\omega}=\mathrm{Cl}(f) / U_{\omega},
\end{gathered}
$$

$k_{\omega}$ is the field corresponding to $U_{\omega}$.

Now, $F$ is constructed by canonical elliptic units $\theta_{\omega}(a)$ of $L\left(a \in A_{\omega}, \omega \in W\right)$. (For elliptic units in the case where $H_{(f)}$ is the ring class field, see [5].) For each class $\omega$ of $W$, we take integers $\lambda_{i a}\left(i=1, \ldots, r_{\omega} ; a \in A_{\omega}\right)$ such that $d_{\omega}=\left|\operatorname{det}\left(v_{i j}\right)\right| \neq 0$ where

$$
v_{i j}=\sum_{a \in A_{\omega}} \lambda_{i a}\left(\left(\chi^{j}(a)-1\right)+\chi^{j}\left(c_{0}\right)\left(\chi^{-j}(a)-1\right)\right) \quad \text { for } i, j=1, \ldots, r_{\omega} .
$$

Let $\theta_{i \omega}=\prod_{a \in A_{\omega}}\left(\theta_{\omega}(a)^{1+\left(c_{0}, L / k\right) J}\right)^{\lambda_{i a}}$. The group $F$ is generated by $\left\{\theta_{i \omega} \mid\right.$ $\left.i=1, \ldots, r_{\omega}, \omega \in W\right\}$. We give the constant $c$ in $(0.1)$ as follows. Let

$$
c_{1}=n^{(r-1) / 2}, \quad c_{2}=n_{0}^{\left(1-r_{0}\right) / 2} \quad \text { and } \quad c_{3}=\prod_{\omega \in W} d_{\omega} m_{\omega}^{r \omega}
$$

where $n_{0}=\left[K_{0}: Q\right]$. Let $c_{4}=c_{1} c_{2} c_{3}$ and $c_{5}=\prod_{\omega \in W} 24 t_{\omega}$, where $t_{\omega}=\min \{t \mid$ $\left.t\left(U_{\omega}: 1\right)=0\left(\bmod h_{k}\right)\right\}, h_{k}$ is the class number of $k$. The constant $c$ is given by $c=c_{4} c_{5}$.

Remark 2. Let $b$ be the number of classes in $W$ which have even order. Using II, Satz 3.2 in [6], we can take $c_{5}=2^{b}$ by the choice of $\theta_{\omega}(a)$. For the number $d_{\omega}$, Schertz [6] showed that we can take $d_{\omega}$ such that $d_{\omega} \neq 0$ and $d_{\omega}$ divides $\left(4 m_{\omega}\right)^{\varphi\left(m_{\omega}\right) / 2} \Phi_{m}(1)\left|D_{m}^{+}\right|$. But this is not enough for our purpose. 
3. Proof of Theorem 1. First, by Remark 2, we can take $c_{5}=2^{b}$ for some integer $b$. Therefore, if we prove $c_{4}=1$ then $c=2^{b}$. Since $L / k$ is cyclic, we assume that $X=\langle\chi\rangle$. Since $L / Q$ is dihedral, $\chi^{J}=\chi^{-1}$. Hence, the relation $\left(\chi^{i}\right)^{J}=\chi^{i}$ implies that the order of $\chi^{i}$ is 1 or 2 . Therefore,

$$
X_{0}= \begin{cases}\left\{1, \chi^{n / 2}\right\} & \text { if } n \text { is even, } \\ \{1\} & \text { if } n \text { is odd }\end{cases}
$$

Therefore, $n_{0}=\left[K_{0}: Q\right]=1$ or 2 . Let

$$
e= \begin{cases}1 & \text { if } n \text { is even, } \\ 0 & \text { if } n \text { is odd }\end{cases}
$$

Then we have $n_{0}=2^{e}$. For the value of $\chi\left(c_{0}\right)$, the next lemma holds.

Lemma 5. Let notations be as above. Let $n=[L: k]$. Assume that $n=2^{a} u,(2, u)=1$. Then there is an element $b$ of $\mathrm{Cl}(f)$ such that $G\left(L / K^{\prime}\right)$ $=\left\langle\left(c_{0} b^{2}, L / k\right) J\right\rangle$ and $\chi\left(c_{0} b^{2}\right)$ is a $2^{a}$-th root of unity. Here, $K^{\prime}$ is a conjugate of $K$.

Proof. Let $(b, L / k) \in G(L / k)$. Then the conjugate of $\left(c_{0}, L / k\right) J$ by $(b, L / k)$ is $\left(b^{-2} c_{0}, L / k\right)$. Since $(u, 2)=1$, we can choose an element $b$ of $\mathrm{Cl}(f)$ such that $\chi\left(b^{-2} c_{0}\right)$ is a $2^{a}$-th root of unity.

By the above lemma, we assume that $\chi\left(c_{0}\right)$ is a $2^{a}$-th root of unity. We shall prove the theorem by considering three cases.

Case 1. $\chi\left(c_{0}\right)=1$. Fix a class $\omega$ of $W$. Since $\chi\left(c_{0}\right)=1, \chi^{j}\left(c_{0}\right)=1$ for any $j$. Therefore $v_{i j}=\sum_{a \in A_{\omega}} \lambda_{i a}\left(\psi^{j}(a)+\psi^{-j}(a)-2\right)$. Put $m=m_{\omega}$ and $r=r_{\omega}$. Since $v_{i j} \in \mathcal{O}^{+}$and $\left\{v_{i j} \mid \psi^{j} \in \omega^{\prime}\right\}$ are all conjugates of $v_{i 1}$ over $\boldsymbol{Q}_{m}^{+} / \boldsymbol{Q}, d_{\omega}=\left|\operatorname{det}\left(v_{i j}\right)\right|$ $=d\left(\left[v_{11}, \ldots, v_{r 1}\right]\right)^{1 / 2}$, where $r=\varphi(m) / 2$. Let $\zeta_{0}=\psi\left(c_{0}\right)$. Then $M$ $=\sum_{j=1}^{m-1} Z\left(\zeta^{j}+\zeta^{-j}-2\right)$. Using Lemmas 1 and 2 , we have

$$
d(M)=\Phi_{m}(1)^{2}\left(m^{\varphi(m)} / \prod_{p \mid m} p^{\Phi(m) /(p-1)} \Phi_{m}(1) \Phi_{m / 2}(1)\right)^{1 / 2} .
$$

Assume that $\left\{v_{11}, \ldots, v_{r 1}\right\}$ is a basis of $M$. Then, from (3.1),

$$
d_{\omega}=\Phi_{m}(1)\left(m^{\varphi(m)} / \prod_{p \mid m} p^{\varphi(m) /(p-1)} \Phi_{m}(1) \Phi_{m / 2}(1)\right)^{1 / 4} .
$$

We shall compute the coefficient $c_{4}$. Since $L / k$ is cyclic, the correspondence between divisors of $n$ and classes of $W$ is one-to-one. So, put $d_{m}=d_{\omega}$. We have $\prod_{\omega \in W} d_{\omega} m_{\omega}^{-r_{\omega}}=\prod_{m \mid n}^{\prime} d_{m} m^{-\varphi(m) / 2}$, where the product is taken over all divisors of $n$ except 1 and 2. From (3.2), we have

$$
\prod_{m \mid n}^{\prime} d_{m} m^{-\varphi(m) / 2}=\prod_{m \mid n}^{\prime} \Phi_{m}(1)^{3 / 4} \prod_{m \mid n}^{\prime}\left(m^{\varphi(m)} \prod_{p \mid m} p^{\varphi(m) /(p-1)} \Phi_{m / 2}(1)\right)^{-1 / 4} .
$$

For any positive integer $n$, the formulas

$$
\sum_{m \mid n} \varphi(m)=n, \quad \prod_{m \mid n} \Phi_{m}(1)=n
$$


are known. By comparison of the index of each prime divisor of $n$, we obtain

$$
\prod_{m \mid n}\left(m^{\varphi(m)} \prod_{p \mid m} p^{\varphi(m) /(p-1)}\right)=n^{n} .
$$

Under the factorization of $n$ in Lemma 5, using (1.1),

$$
\prod_{m \mid n} \Phi_{m / 2}(1)=\prod_{m \mid u} \Phi_{m}(1)^{e} \prod_{m \mid 2} \Phi_{m / 2}(1)=(n / 2)^{e} .
$$

Using (3.3)-(3.6),

$$
\prod_{m \mid n}^{\prime} d_{m} m^{-\varphi(m) / 2}=n^{(3-e-n) / 4} .
$$

Since $L / Q$ is cyclic, using (2.1), the rank $r$ of $E$ is given by

$$
r-r_{0}=\left(\sum_{m \mid n} \varphi(m)-\varphi(2)-\varphi(1)\right) / 2 .
$$

By the assumption on the degree $n, \chi\left(c_{0}\right)=1$ yields that

$$
\begin{cases}r_{0}=0, K_{0}=Q & \text { if } n \text { is odd, } \\ r_{0}=1, K_{0} \text { is a real quadratic field } & \text { if } n \text { is even. }\end{cases}
$$

We obtain $r_{0}=e$. From (3.4) and (3.8), the rank of $E$ is

$$
r=(n-1-e) / 2 \text {. }
$$

From the definition of the constants $c_{1}, c_{2}, c_{3}$ in Section 2, we have

$$
c_{1}=n^{(r-1) / 2}=n^{(n-3+e) / 4} .
$$

Since $r_{0}=e=0$ or 1 , and $n_{0}=2^{e}$,

$$
c_{2}=2^{e(1-e) / 2}=1 \text {. }
$$

From (3.7), $c_{3}=n^{(3-e-n) / 4}$. Hence $c_{4}=c_{1} c_{2} c_{3}=1$. In this case, the theorem is proved.

Case 2. $\chi\left(c_{0}\right)=-1$. If $m_{\omega}$ is odd then $d_{\omega}$ is the same as in case 1 . Therefore we shall compare cases 1 and 2 for the value of $d_{\omega}$ only in the case where $m_{\omega}$ is even. For that purpose, we write $c_{1}^{\prime}, d_{\omega}^{\prime}$ instead of $c_{1}, d_{\omega}$ in case 1 and so on. If $(j, n)=1$, then $j$ is odd and $\chi^{j}\left(c_{0}\right)=-1$. Therefore,

$$
v_{i j}=\sum_{a \in A_{\omega}} \lambda_{i a}\left(\psi^{j}(a)-\psi^{-j}(a)\right) \quad \text { and } \quad v_{i j} \in M=\sum_{j=1}^{m-1} Z\left(\zeta^{j}-\zeta^{-j}\right) .
$$

Suppose that $\left\{v_{11}, \ldots, v_{r 1}\right\}$ is a basis of $M$. Then using Lemma 3,

$$
d_{\omega}=\left(\Phi_{m}(1) \Phi_{m}(-1)\right)^{1 / 2}\left|D_{m}^{+}\right|^{1 / 2} \text {. }
$$

By comparison of (3.2) and (3.9),

$$
d_{\omega}^{\prime} / d_{\omega}=\Phi_{m}(1) /\left(\Phi_{m}(1) \Phi_{m}(-1)\right)^{1 / 2} .
$$


Put $m=2^{a} u,(2, u)=1$, where $a \geqslant 1$. By the assumption of the degree $n$, if $a \geqslant 2$ then $n=4$ and $\Phi_{m}(1)=\Phi_{m}(-1)=2$. If $a=1$ then $\Phi_{m}(1) \doteq 1$. Therefore,

$$
d_{\omega}^{\prime} / d_{\omega}= \begin{cases}\Phi_{m}(-1)^{-1 / 2} & \text { if } a=1, \\ 1 & \text { otherwise. }\end{cases}
$$

Assume $a>1$. Then $d_{\omega}^{\prime} / d_{\omega}=1$. Since $\chi^{2}\left(c_{0}\right)=1, r_{0}=1$. Therefore, $c_{4}=c_{4}^{\prime}=1$. Assume $a=1$. Then $d_{\omega}^{\prime} / d_{\omega}=\Phi_{m}(-1)^{1 / 2}$. Therefore, $\prod_{\omega \in W}\left(d_{\omega}^{\prime} / d_{\omega}\right)=u^{1 / 2}$. Since $r_{0}=0$, the rank $r=r^{\prime}-1$. Then $c_{1}^{\prime} c_{2}^{\prime} / c_{1} c_{2}=(n / 2)^{1 / 2}$. Therefore $c_{4}^{\prime} / c_{4}=1$. In this case, the theorem is proved.

Case 3. $\chi\left(c_{0}\right) \neq \pm 1$. In this case, by the assumption of $n$, we have $n=4$ and $W=\{\chi\}$. We compute $d_{\omega}$ immediately using Lemma 4 . Then $d_{\omega}=2^{3 / 2}$. Since $n_{0}=2, r_{0}=0$ and $r=1$. So $c_{4}=1$. Now, the proof of Theorem 1 is complete.

COROllary. Let $L$ be cyclic over $k$. If the maximal real subfield $K$ of $L$ is non-Galois over $\boldsymbol{Q}$ then

$$
h=2^{b} h_{0}\left(E: F E_{0}\right) \text {. }
$$

Proof. Since $K$ is the maximal real subfield of $L, \chi\left(c_{0}\right)=1$. Therefore, the corollary can be proved as in the above proof of case 1 .

4. Non-dihedral cases. In this section, we consider the case where $n=p$ or $n=p q$, both $p$ and $q$ are primes, and the case is not included in Theorem 1. We consider the automorphism $\tau$ of order 2 instead of the complex conjugation $J$ on $X$. When $\left\langle\chi^{\tau}\right\rangle \neq\langle\chi\rangle$, we choose representatives of the class $\omega$ of $W$ and give $v_{i j}$ 's which are different from those of Section 2. (More details in [6].) If $n=p$, then $L$ has only one character class, therefore, $L / Q$ is dihedral. Suppose $n=p q$. Similarly, if $p=2$ and $q \neq 2$ then $L / Q$ is dihedral. If $p=q=2$ then $L / Q$ is dihedral because $G(L / Q)$ is a non-abelian group of order 8 . Suppose that both $p$ and $q$ are odd primes. Then the next theorem holds.

THEOREM 2. Let $K / Q$ be non-Galois and suppose the Galois closure $L$ of $K$ is abelian over $k$. Suppose $n=[K: Q]=p q$ (both $p$ and $q$ are odd primes), and $L / Q$ is not dihedral. Then we can construct a group $F$ of elliptic units such that if $p=q$ then $G(L / k)$ is an abelian group of type $(p, p)$ and,

$$
p^{\left(p^{2}+2 p-3\right) / 4} h / h_{0}=\left(E: F E_{0}\right)
$$

if $p \neq q$ and $K_{0} \neq Q$ then,

$$
2^{(p-1)(q-1) / 2} h / h_{0}=\left(E: F E_{0}\right) \text {. }
$$

Proof. In both cases above, the character group $X$ is the direct product $\langle\chi\rangle \times\langle\psi\rangle$ where $\chi$ and $\psi$ are the characters of order $p$ and $q$, respectively. Since $\tau^{2}=1$ and $L / Q$ is not abelian, we assume that $\psi^{\tau} \neq \psi^{-1}$. We denote by $\langle\psi\rangle^{*}$ the subset of $\langle\psi\rangle$ whose element has order $m$ where $m$ is the order of $\psi$. Let $p=q$. Then we have two cases: (1) $\chi^{\tau}=\chi^{-1}$, (2) $\chi^{\tau}=\chi$. 
Case 1: $W$ has $p+1$ classes $\langle\chi\rangle^{*}$ and $\left\langle\chi^{i} \psi\right\rangle^{*}(i=1, \ldots, p)$. In this case, the construction of $F$ is the same as in Theorem 1. For any class $\omega$, $d_{\omega}=p^{(p+1) / 4}$ and $r_{\omega}=\varphi(p) / 2$. Therefore $c_{4}=p^{\left(p^{2}+2 p-3\right) / 4}$.

Case 2: $X_{0}=\langle\chi\rangle .\left\langle\left(\psi^{i} \chi\right)^{\tau}\right\rangle=\left\langle\psi^{-i} \chi\right\rangle \neq\left\langle\psi^{i} \chi\right\rangle(i=1, \ldots,(p-1) / 2)$. Then $W$ has $(p+1) / 2$ classes $\langle\psi\rangle^{*}$ and $\left\langle\psi^{i} \chi\right\rangle^{*} \cup\left\langle\psi^{-i} \chi\right\rangle^{*}(i=1, \ldots,(p-1) / 2)$. If $\omega=\langle\psi\rangle^{*}$ then $d_{\omega}$ is the same as in case 1 . Otherwise, we take $v_{i j}$ in Section 2 as

$$
v_{i j}=\sum_{a \in A_{\omega}} \lambda_{i a}\left(\phi^{j}(a)-1\right)
$$

for $i, j=1, \ldots, p$ where $\phi \in\left\langle\psi^{k} \chi\right\rangle^{*}$ for some $k$. Then $m_{\omega}=p$ and $d_{\omega}$ is the discriminant of the $Z$-module constructed by $v_{i j}$ 's. We can take $d_{\omega}=\Phi_{p}(1)\left|D_{p}\right|^{1 / 2}$ in [6], I, Satz 1.4. Therefore, $c_{4}=\prod_{\omega \in W} d_{\omega} m_{\omega}^{-r_{\omega}}=p^{\left(p^{2}+2 p-3\right) / 4}$ where the product is taken over all classes of $W$. In the former case, the theorem is proved.

Let $p \neq q$. Then $X_{0}=\langle\chi\rangle$. Since $L / k$ is cyclic, $W$ has two classes $\langle\chi\rangle^{*}$ and $\langle\chi \psi\rangle^{*}$. If $\omega=\langle\chi\rangle^{*}$ then $d_{\omega}$ is the same as in Theorem 1. Let $Q_{p q}^{*}$ be the fixed field of $Q_{p q}$ for $\tau$. Let $\omega=\langle\chi \psi\rangle^{*}$ and $M$ be the $Z$-module constructed by $v_{i j}$ in Section 2. Then $M$ includes the $Z$-module $2\left(p \cap \mathcal{O}^{*}\right)$ where $\mathcal{O}^{*}$ is the ring of integers of $Q_{p q}^{*}$. The discriminant $D^{*}$ of $\mathcal{O}^{*}$ is $(p q)^{\varphi(p q) / 2} q^{1-p}$ which is easily shown by examining the ramification of $\boldsymbol{Q}_{p q} / \boldsymbol{Q}_{p q}^{*}$. If we take $v_{i j}$ as a basis of $2\left(p \cap \mathcal{O}^{*}\right)$, then $d_{\omega}=2^{\varphi(p q) / 2} \Phi_{p q}(1)\left|D^{*}\right|^{1 / 2}$. Therefore, $c_{4}=2^{(p-1)(q-1) / 2}$. The proof is completed.

EXAmple. Let $K_{0}$ be abelian of degree $p$. Let $K \supset K_{0}$ and $K / Q$ be non-Galois of degree $p q$. Suppose the Galois closure $L$ of $K$ to be abelian over an imaginary quadratic field $k$ and $[L: k]=p q$. Then $L / Q$ is not dihedral.

\section{References}

[1] H. Hay ashi, On elliptic units and class number of a certain dihedral extension of degree $2 l$, Acta Arith. 44 (1985), 35-45.

[2] K. Na k a m u la, Class number calculation of a cubic field from the elliptic unit, J. Reine Angew. Math. 331 (1982), 114-123.

[3] - Class number calculation of a quartic field from the elliptic unit, Acta Arith. 45 (1985), 215-227.

[4] - Class number calculation of a sextic field from the elliptic unit, ibid., 229-247.

[5] R. Schertz, Arithmetische Ausdeutung der Klassenzahlformel für einfach reele kubische Zahlkörper, Abh. Math. Sem. Univ. Hamburg 41 (1974), 221-223.

[6] - Die Klassenzahl der Teilkörper abelscher Erweiterungen imaginär-quadratischer Zahlkörper, I, J. Reine Angew. Math. 295 (1977), 151-168; II, ibid. 296 (1977), 58-79.

[7] L. C. Washingt on, Introduction to Cyclotomic Fields, Springer-Verlag, New York-Heidelberg-Berlin 1982. 